\title{
Null Association between BRAF V600E Mutation and Tumor Recurrence in Patients with Papillary Thyroid Microcarcinoma in South Korea
}

\author{
Ji Yoon Kim ${ }^{1}$, Kyoung Jin Kim${ }^{1}$, Jae Hyun Bae ${ }^{1}$, Joo Hyung Kim${ }^{1}$, \\ Nam Hoon Kim ${ }^{1}$, Hee Young Kim ${ }^{1}$, Hoon Yub Kim², Seung-Kuk Baek ${ }^{3}$, \\ Sin Gon Kim ${ }^{1}$, Kwang Yoon Jung ${ }^{3}$ and Kyeong Jin Kim ${ }^{1}$
}

Division of Endocrinology and Metabolism ${ }^{1}$, Department of Surgery'2, Department of Otorhinolaryngology-Head and Neck Surgery ${ }^{3}$, Korea University College of Medicine, Seoul, Korea

\begin{abstract}
Background and Objectives: The clinical implications of the BRAF V600E mutation in papillary thyroid microcarcinoma (PTMC), defined as $\leq 1.0 \mathrm{~cm}$ of tumor size, remain controversial. We investigated the association between the BRAF V600E mutation and PTMC recurrence in a retrospective cohort of patients with thyroid cancer. Materials and Methods: This study included 2319 patients with PTMC (median age, 50 years [interquartile range (IQR), 41-57 years]) who underwent thyroid surgery from 2010 to 2019 at a single tertiary medical center. The median follow-up time was 75 months (IQR, 30-98 months). Tumor recurrence was confirmed by histological, cytological, radiographic, and biochemical criteria, combined with persistent and recurrent disease. Results: A total of $60.2 \%$ (1395/2319) patients with PTMC had the BRAFV600E mutation. The tumor recurrence rate was $2.1 \%$ (19/924) in BRAF mutation-negative patients and 2.9\% (41/1395) in BRAF mutation-positive patients, with a hazard ratio (HR) of 1.05 (95\% confidence interval [Cl], 0.61-1.84) after adjusting for clinicopathological risk factors. Similar results were found in patients with high-risk PTMC (adjusted HR, 1.09; 95\% Cl, 0.56-2.11) who had lymph node metastasis (LNM), extrathyroidal extension (ETE), or distant metastasis (DM) at diagnosis and in patients with low-risk PTMC (adjusted HR, 1.00; $95 \% \mathrm{Cl}, 0.35-2.83$ ) who had no LNM, ETE, or DM. Conclusion: The finding that the BRAF V600E mutation was not associated with tumor recurrence in our cohort of Korean patients with PTMC, especially in patients with low-risk PTMC, suggests that its value in the prediction of disease progression is limited.
\end{abstract}

Key Words: Papillary thyroid microcarcinoma, BRAF V600E mutation, Active surveillance, Tumor recurrence

\section{Introduction}

Papillary thyroid microcarcinoma (PTMC), defined as a tumor that is not greater than $1 \mathrm{~cm}$ in its greatest dimension, accounts for approximately $50 \%$ of differentiated thyroid cancer (DTC) cases. $^{1-3)}$ Moreover, previous studies have shown a robust increase in
PTMC incidence, with an average annual increase of $9.3 \% .^{3,4)}$

Despite the clinical importance of PTMC, controversies over its treatment have not been clearly resolved since active surveillance (AS) has been endorsed by the American Thyroid Association (ATA) and Korean Thyroid Association (KTA) guidelines. ${ }^{5,6}$ The crux of this debate is whether very low-risk PTMC,

Received September 8, 2021 / Revised November 7, 2021 / Accepted November 9, 2021

Correspondence: Kyeong Jin Kim, MD, PhD, Division of Endocrinology and Metabolism, Department of Internal Medicine, Korea University College of Medicine, Korea University Anam Hospital, 73, Goryeodae-ro, Seongbuk-gu, Seoul 02841, Korea Tel: 82-2-920-6285, Fax:82-2-953-9355, E-mail: jins0707@korea.ac.kr 
with no clinical evidence of metastasis and local invasion, is indeed indolent and whether a novel approach including genetic markers can predict poor prognosis in this group.

In the past decades, the BRAF V600E mutation has been widely investigated as a prognostic marker in DTC, suggesting that BRAF mutation is related with tumor recurrence as well as aggressive clinicopathological characteristics. ${ }^{7,8)}$ However, there have been limited and discordant results on the clinical significance of BRAF mutation in PTMC. Chen et al. ${ }^{9)}$ have demonstrated that BRAF mutation correlates with tumor recurrence in PTMC using multi-institutional data and meta-analysis. Choi et al., ${ }^{10)}$ in contrast, did not find a significant association between BRAF mutations and aggressive clinicopathological factors in PTMC. These inconsistent results can be explained by differences in sample size, ethnicity, length of follow-up, and BRAF mutation prevalence, leaving this issue open to further debate. Moreover, the prognostic importance of BRAF mutations in low-risk PTMC cases considered candidates for non-surgical AS has not been clearly elucidated, particularly in areas where the BRAF mutation is prevalent.

In this context, we investigated the prognostic implication of BRAF V600E for tumor recurrence in PTMC, especially for low-risk PTMC, in South Korea, where the BRAF mutation is highly prevalent.

\section{Materials and Methods}

\section{Study Design and Patients}

This retrospective study initially analyzed patients from the Korea University Anam Hospital Thyroid Cancer Cohort, which included 6256 patients treated for papillary thyroid cancer at the Korea University Anam Hospital in Seoul, Korea since 2010. This database contains information on treatment modalities, tumor recurrence, and basic clinicopathological and demographic characteristics. This study was approved by the Institutional Review Board (IRB) of the Korea University Anam Hospital (IRB No. 2021AN0297). The requirement for informed consent was waived due to the retrospective study design, and all patient data were anonymized and de-identified. From the original cohort, we selected 2319 patients with BRAF V600E mutation status who had been confirmed to have PTMC by surgical resection. Fig. 1 shows a schematic diagram of the study flow.

\section{Definition of Low- and High-Risk PTMC and Tumor Recurrence}

Low-risk PTMC was defined as PTMC with no extrathyroidal extension (ETE), cervical lymph node metastasis (LNM), and distant metastasis (DM), while high-risk PTMC was defined as PTMC with at least one of these features. All patients underwent hemithyroidectomy or total thyroidectomy. Cervical lymph node dissection and radioactive iodine ablation (RAI) were performed when clinically indicated. The primary outcome was tumor recurrence defined as persistent or recurrent disease, confirmed by histological, cytological, radiographic, and biochemical criteria. ${ }^{5,6,11)}$ All patients were followed up until the earliest occurrence of tumor recurrence, death, or the end of the study period (December 31, 2020). For BRAF V600E mutation analyses, we amplified exon 15 of the BRAF gene using the PNAClamp BRAF Mutation Detection Kit (Panagene Ltd., Daejeon, Korea) based on a peptide nucleic acid (a synthetic DNA analog)-mediated PCR clamping technique. This approach has shown high sensitivity and specificity with a small amount of DNA,

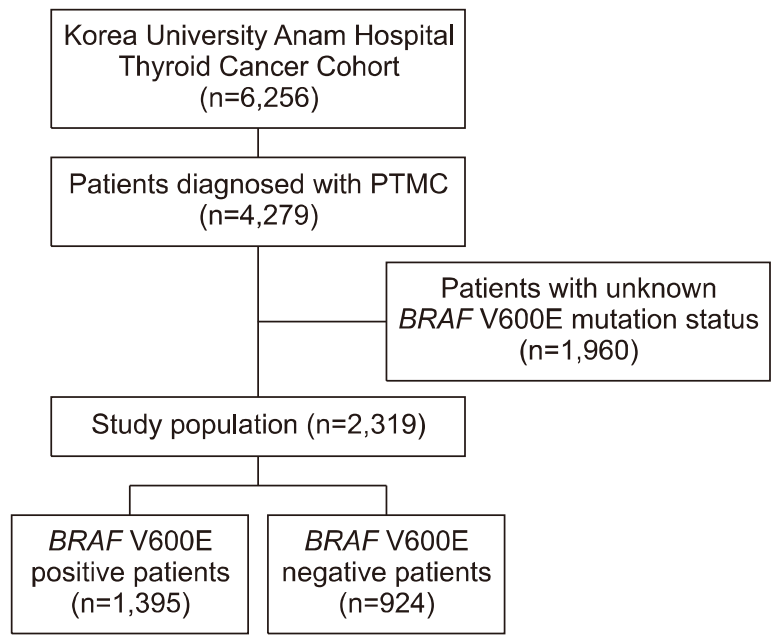

Fig. 1. Flow diagram of the study subject selection process. 
and the details of the procedures have been described previously. ${ }^{12,13)}$ The physician was informed of the patient's BRAF mutation status after surgery.

\section{Statistical Analysis}

Continuous data of non-normally distributed variables are presented as medians and interquartile ranges (IQR), using the Wilcoxon-Mann-Whitney test, and categorical data are presented as numbers and percentages, using a chi-square test or Fisher's exact test. For tumor recurrence, we calculated the hazard ratios (HRs) with 95\% confidence intervals (Cls) using Cox proportional hazard regression models. These analyses were performed according to the BRAF mutation status and further adjusted for patient age at diagnosis, sex, tumor size, LNM, ETE, multifocality, surgery type, and RAl treatments. To analyze recurrence-free survival, Kaplan-Meier survival curves with log-rank tests were used.

All statistical analyses were performed using SPSS version 23 (IBM SPSS Inc. New York, NY, USA) and $R$ version 4.0.2 (R Foundation for Statistical Computing, www.R-project.org). Graphs were generated using
GraphPad Prism version 9 (GraphPad Software, San Diego, CA, USA). Statistical significance was set at a two-sided $p$-value of $<0.05$.

\section{Results}

\section{Clinicopathological Characteristics of PTMC Based on the BRAF V600E Mutation Status}

Table 1 presents the baseline characteristics of all patients with PTMC $(n=2319)$. The median age of patients was 50 years (IQR, 41-57 years), and $82.2 \%$ (1907/2319) were women. The overall prevalence of the BRAF V600E mutation was 60.2\% (1395/2319). The median tumor size was $0.6 \mathrm{~cm}$ for BRAF mutation-positive cases and $0.5 \mathrm{~cm}$ for $B R A F$ mutation-negative cases $(p<0.001)$. BRAF mutations were significantly associated with LNM (23.3\% vs. 31.0\%, $\mathrm{p}<0.001$ for BRAF mutation-negative and -positive cases, respectively). However, BRAF mutations were not significantly associated with gross ETE, vascular invasion, or multifocality. Total thyroidectomy and RAI therapy were performed in $60.9 \%(n=1412)$ and $37.6 \%$

Table 1. Clinicopathological demographic characteristics of papillary thyroid microcarcinoma according to BRAF genotype

\begin{tabular}{|c|c|c|c|c|}
\hline & All patients & $\begin{array}{c}\text { BRAF V } 600 \mathrm{E} \text {-negative } \\
\text { patients }\end{array}$ & $\begin{array}{c}\text { BRAF V600E-positive } \\
\text { patients }\end{array}$ & $p$ value \\
\hline Total cases, $\mathrm{n}(\%)$ & 2319 & $924(39.8)$ & $1395(60.2)$ & \\
\hline Age, median (IQR), years & $50(41-57)$ & $50(41-58)$ & $49(40-57)$ & 0.062 \\
\hline Female sex, $\mathrm{n}(\%)$ & $1907(82.2)$ & $773(83.7)$ & $1134(81.3)$ & 0.144 \\
\hline Tumor size, median (IQR), cm & $0.6(0.4-0.8)$ & $0.5(0.4-0.7)$ & $0.6(0.5-0.8)$ & $<0.001$ \\
\hline Extrathyroidal extension, n (\%) & $23(1.0)$ & $11(1.2)$ & $12(0.9)$ & 0.432 \\
\hline Lymph node metastasis, $\mathrm{n}(\%)$ & $648(27.9)$ & $215(23.3)$ & $433(31.0)$ & $<0.001$ \\
\hline Vascular invasion, $\mathrm{n}(\%)$ & $6(0.3)$ & $1(0.1)$ & $5(0.4)$ & $0.412^{*}$ \\
\hline Multifocality, n (\%) & $899(38.8)$ & $351(38.0)$ & $548(39.3)$ & 0.531 \\
\hline Distant metastasis, n (\%) & $1(0.04)$ & $0(0.0)$ & $1(0.1)$ & $1.00 *$ \\
\hline Stage (the AJCC $\left.8^{\text {th }}\right), \mathrm{n}(\%)$ & & & & $0.238 *$ \\
\hline l & $2126(91.7)$ & $851(92.1)$ & $1275(91.4)$ & \\
\hline$\|$ & $190(8.2)$ & $71(7.7)$ & $119(8.5)$ & \\
\hline III & $2(0.1)$ & $2(0.2)$ & $0(0.0)$ & \\
\hline IV & $1(0.04)$ & $0(0.0)$ & $1(0.1)$ & \\
\hline Total thyroidectomy, $\mathrm{n}(\%)$ & $1412(60.9)$ & $530(57.4)$ & $882(63.2)$ & 0.005 \\
\hline${ }^{131}$ I treatment, $\mathrm{n}(\%)$ & $873(37.6)$ & $306(33.1)$ & $567(40.6)$ & $<0.001$ \\
\hline${ }^{131}$ I dose, median (IQR), mCi & $100(30-150)$ & $100(30-150)$ & $100(30-150)$ & 0.635 \\
\hline Follow time, median (IQR), months & $75(30-98)$ & $75(33-99)$ & $75(27-97)$ & 0.222 \\
\hline Tumor recurrence, $\mathrm{n}(\%)$ & $60(2.6)$ & $19(2.1)$ & $41(2.9)$ & 0.190 \\
\hline
\end{tabular}

AJCC: American Joint Committee on Cancer, IQR: interquartile range

${ }^{*}$ Calculated using Fisher's exact test. 
( $n=873$ ) of the total number of patients, respectively. Patients with BRAF mutations underwent total thyroidectomies and RAI treatment more frequently than those without BRAF mutations.

\section{Effect of BRAF V600E Mutation on PTMC Recurrence}

During the median follow-up period of 75 months (IQR, 30-98 months), tumor recurrence occurred in $2.6 \%(n=60)$ patients. The recurrence rate was $2.9 \%$ (41/1395) and 2.1\% (19/924) for BRAF mutation-positive and -negative patients, respectively (Table 2), with an unadjusted $\mathrm{HR}$ of $1.48(95 \% \mathrm{Cl}, 0.86-2.55)$. After adjustments for age, sex, tumor size, LNM, ETE, multifocality, surgical type, and RAl treatments, the effect of the BRAF mutation on tumor recurrence remained insignificant, with an $\mathrm{HR}$ of $1.05(95 \% \mathrm{Cl}$, 0.61-1.84). The Kaplan-Meier survival curve also showed no significant difference between BRAF mutation-positive and -negative patients in regard to recurrence-free survival (log-rank, $p=0.157$; Fig. 2A).

We also evaluated whether BRAF V600E mutation affected tumor recurrence differently in low-risk and high-risk groups. The overall recurrence rates were 1.0\% (16/1662) for low-risk PTMC and 6.7\% (44/657) for high-risk PTMC. The BRAF V600E mutation did not significantly affect tumor recurrence in either low-risk or high-risk PTMC (Table 2). For low-risk PTMC, the recurrence rates were $0.9 \%(6 / 703)$ in BRAF mutation-negative patients and $1.0 \%(10 / 959)$ in BRAF mutation-positive patients, with an adjusted HR of 1.00 (95\% Cl, 0.35-2.83). For high-risk PTMC, the recurrence rates were $5.9 \%(13 / 221)$ and $7.1 \%(31 / 436)$ in BRAF mutation-negative and -positive patients, respectively, with an adjusted HR of $1.09(95 \% \mathrm{Cl}$, 0.56-2.11). Similar results were obtained using KaplanMeier analyses (Fig. 2B, C). In both groups, the BRAF V600E mutation had no significant effect on recurrence-free survival (log-rank $p=0.611$ for low-risk PTMC and log-rank $p=0.606$ for high-risk PTMC).

\section{Effect of Other Clinicopathological Factors on PTMC Recurrence}

Univariate and multivariate Cox proportional analyses were performed to investigate the risk factors for tumor recurrence (Table 3). Conventional risk factors such as male sex, LNM, ETE, tumor size and multifocality affected tumor recurrence in univariate analysis, and especially male sex (adjusted HR, 2.25; 95\% $\mathrm{Cl}$, 1.25-4.10), LNM (adjusted HR, 5.62; $95 \% \mathrm{Cl}$, 2.88-10.99) and multifocality (adjusted HR, 2.43; 95\% $\mathrm{Cl}, 1.33-4.44)$ remained significant in multivariate analysis.

Table 2. Hazard ratios of the BRAF V600E mutation for PTMC recurrence

\begin{tabular}{|c|c|c|c|c|c|}
\hline & \multirow{2}{*}{ Recurrence, n (\%) } & \multicolumn{2}{|c|}{ Unadjusted } & \multicolumn{2}{|c|}{ Adjusted } \\
\hline & & $\mathrm{HR}(95 \% \mathrm{Cl})$ & $p$ value & $\mathrm{HR}(95 \% \mathrm{Cl})$ & $p$ value \\
\hline \multicolumn{6}{|l|}{ All PTMCs } \\
\hline BRAF V600E-negative & $19 / 924(2.1)$ & 1 (reference) & & 1 (reference) & \\
\hline BRAF V600E-positive & $41 / 1395(2.9)$ & $1.48(0.86-2.55)$ & 0.159 & $1.05(0.61-1.84)$ & $0.850 *$ \\
\hline \multicolumn{6}{|l|}{ Low-risk PTMC } \\
\hline BRAF V600E-negative & $6 / 703(0.9)$ & 1 (reference) & & 1 (reference) & \\
\hline BRAF V600E-positive & $10 / 959(1.0)$ & $1.30(0.47-3.60)$ & 0.612 & $1.00(0.35-2.83)$ & $0.999^{\dagger}$ \\
\hline \multicolumn{6}{|l|}{ High-risk PTMC } \\
\hline BRAF V600E-negative & $13 / 221(5.9)$ & 1 (reference) & & 1 (reference) & \\
\hline BRAF V600E-positive & $31 / 436(7.1)$ & $1.19(0.62-2.27)$ & 0.607 & $1.09(0.56-2.11)$ & $0.802^{\dagger}$ \\
\hline
\end{tabular}

$\mathrm{Cl}$ : confidence interval, HR: hazard ratio, PTMC: papillary thyroid microcarcinoma

${ }^{*}$ Adjusted for patient age at diagnosis (analyzed as a continuous variable), sex (male/female), tumor size (analyzed as a continuous variable), cervical lymph node metastasis (present/absent), extrathyroidal extension (present/absent), multifocality (multifocal/single), surgery type (total thyroidectomy/hemithyroidectomy), and radioactive iodine treatment (received/not received).

${ }^{\dagger}$ Adjusted for patient age at diagnosis, sex, tumor size, multifocality, surgery type, and radioactive iodine treatment. 
A

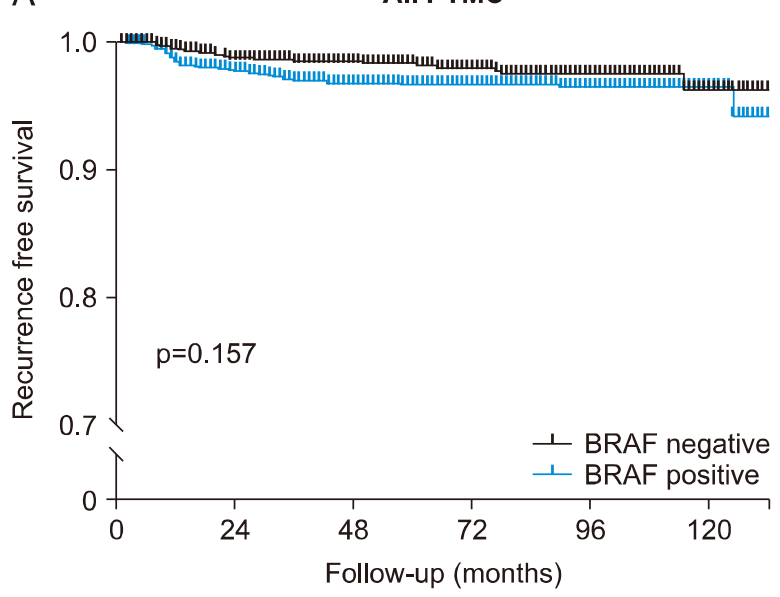

C

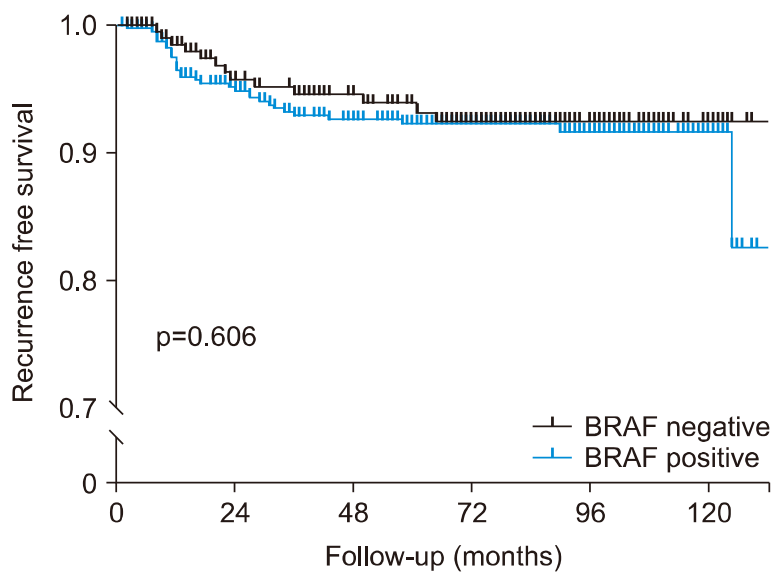

B

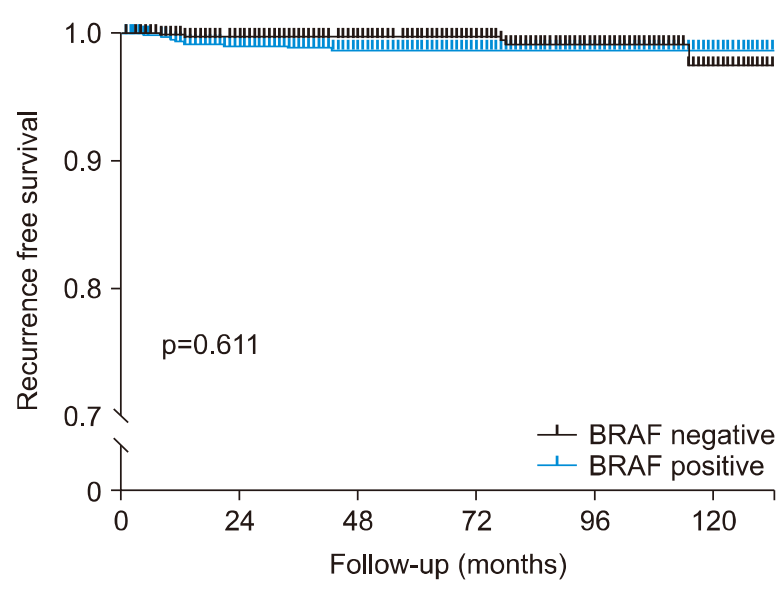

Fig. 2. Kaplan-Meier analysis of disease recurrence-free survival based on BRAF V600E mutation status in papillary thyroid microcarcinoma (PTMC). (A) All types of PTMC. (B) Low-risk PTMC. (C) High-risk PTMC. Followup time is truncated to 132 months.

Table 3. Univariate and multivariate analyses of clinicopathological factors and PTMC recurrence

\begin{tabular}{lccccr} 
& \multicolumn{2}{c}{ Univariate analysis } & & \multicolumn{2}{c}{ Multivariate analysis } \\
\cline { 2 - 3 } \cline { 5 - 6 } & $H R(95 \% \mathrm{Cl})$ & $\mathrm{p}$ value & & HR $(95 \% \mathrm{Cl})$ & $\mathrm{p}$ value \\
\hline Age $\geq 55$ years & $0.73(0.41-1.31)$ & 0.288 & & $0.78(0.41-1.47)$ & 0.443 \\
Male sex & $2.19(1.26-3.81)$ & 0.006 & & $2.25(1.25-4.10)$ & 0.007 \\
Tumor size $>0.5 \mathrm{~cm}$ & $3.26(1.74-6.15)$ & $<0.001$ & & $1.63(0.84-3.17)$ & 0.148 \\
LNM & $7.06(3.98-12.51)$ & $<0.001$ & & $5.62(2.88-10.99)$ & $<0.001$ \\
ETE & $6.59(1.60-27.16)$ & 0.009 & & $1.58(0.21-11.64)$ & 0.656 \\
Multifocality & $2.70(1.60-4.57)$ & $<0.001$ & & $2.43(1.33-4.44)$ & 0.004 \\
BRAF mutation & $1.48(0.86-2.55)$ & 0.159 & & $1.33(0.71-2.49)$ & 0.366 \\
\hline
\end{tabular}

$\mathrm{Cl}$ : confidence interval, ETE: extrathyroidal extension, HR: hazard ratio, LNM: cervical lymph node metastasis, PTMC: papillary thyroid microcarcinoma, RAl: radioactive iodine ablation

All variables were analyzed as categorical values: age $(\geq 55 /<55$ years), sex (male/female), tumor size $(>0.5 / \leq 0.5 \mathrm{~cm})$, LNM (present/absent), ETE (present/absent), multifocality (multifocal/single), and BRAF mutation (positive/negative).

\section{Discussion}

In this retrospective, large, single-center study, we confirmed that patients with PTMC usually have an excellent prognosis, with a tumor recurrence rate of
$2.6 \%$ over a median follow-up period of 75 months. Although patients with BRAF mutation-positive PTMC had numerically higher tumor recurrence rates, when separated into high- and low-risk groups as well as overall, HRs were not statistically significant after ad- 
justing for clinicopathological factors in each group. On the other hand, conventional risk factors such as male sex, LNM, and multifocality were significantly associated with tumor recurrence in PTMC. These results suggest that the BRAF mutation has a limited prognostic value regarding tumor recurrence in PTMC, particularly for low-risk PTMC in Korea, a representative BRAF mutation-prevalent area.

According to the KTA guidelines, PTMC can be observed without immediate surgical treatment if the tumor has no aggressive cytologic features and shows no local/distant metastasis. ${ }^{6}$ However, all PTMC cases may not consistently have a good prognosis in real practice, and these recommendations are based on a few observational studies conducted in the same country. ${ }^{14,15)}$ Hence, there is an increasing need for a prognostic marker to facilitate decision making on the AS of PTMC, and the BRAF V600E mutation can potentially play a decisive role in risk stratification. Several studies have reported that BRAF mutation is associated with tumor recurrence in patients with PTMC, ${ }^{9)}$ especially in those with low-risk PTMC. ${ }^{16)}$ In contrast, studies have shown that the BRAF mutation alone has no independent prognostic effect in PTMC cases. ${ }^{10,17,18)}$ Hence, clinical guidelines on the management of DTC have defined all intrathyroidal PTMCs as a low-risk group, regardless of the BRAF mutation status. ${ }^{5,6)}$ The discrepancy between the results of previous studies may be mainly attributed to the differences in the prevalence of BRAF mutations in each region, ranging from $30 \%$ to $80 \%$ of all PTMCs. $5,10,16,17,19-22)$ In particular, Korea, where our study was conducted, has a higher prevalence of BRAF mutations than most Western countries. ${ }^{10,18,22-24)}$ Two important questions need to be addressed in this regard-the first is why the prevalence of BRAF mutation varies by geographical region, and the second is why the prognostic effect of $B R A F$ mutation seems to be less powerful in areas where BRAF mutation is prevalent. Although these questions remain unresolved, previous studies have reported that high iodine intake is associated with a high prevalence of BRAF mutation, ${ }^{25,26)}$ therefore, we speculate that high iodine intake may influence the negative effects of BRAF mutation on the io- dine metabolism. ${ }^{27,28)}$ Further investigations are needed to answer these questions.

This study has several limitations to be mentioned. First, this was a single-center study, and this study type often requires external validity; however, we included a large number of patients $(n=2319)$ with PTMC. Second, considering the good prognosis of PTMC, ${ }^{14,29,30)}$ relatively short follow-up periods inevitably led to a low disease recurrence rate and reduced the power to conclude firm results. Third, other genetic mutations, such as rat sarcoma mutations and telomerase reverse transcriptase promoter mutations were not analyzed due to a lack of relevant data. Lastly, since the mutation analyses were performed after the surgical treatment and reported to the physician, RAl treatment may have been influenced by the BRAF mutation results.

In summary, this study investigated the prognostic role of the BRAF V600E mutation in PTMC, including low-risk PTMC considered for conservative surveillance instead of immediate surgical treatment, using a large, retrospective, single-center database. An excellent prognosis was observed in PTMC, and the BRAF V600E mutation was not associated with disease recurrence in PTMC, suggesting that the BRAF mutation has limited value as a deciding factor for AS in an area where the mutation is prevalent. Our results would require further investigation into prognostic factors that determine AS in appropriate low-risk PTMC.

\section{Acknowledgments}

We thank the participants of the Korea University Anam Hospital Thyroid Cancer Cohort.

\section{Conflicts of Interest}

No potential conflict of interest relevant to this article was reported.

\section{Orcid}

Ji Yoon Kim: https://orcid.org/0000-0001-6626-2124

Kyoung Jin Kim: https://orcid.org/0000-0001-7925-2515 
Jae Hyun Bae: https://orcid.org/0000-0002-1384-6123 Joo Hyung Kim: https://orcid.org/0000-0002-1704-7943 Nam Hoon Kim: https://orcid.org/0000-0002-9926-1344 Hee Young Kim: https://orcid.org/0000-0002-7268-2624 Hoon Yub Kim: https://orcid.org/0000-0002-6731-3912 Seung-Kuk Baek: https://orcid.org/0000-0002-4751-0337 Sin Gon Kim: https://orcid.org/0000-0002-7430-3675 Kwang Yoon Jung: https://orcid.org/0000-0003-4316-0779 Kyeong Jin Kim: https://orcid.org/0000-0002-5878-6005

\section{References}

1) Davies L, Welch HG. Increasing incidence of thyroid cancer in the United States, 1973-2002. JAMA 2006;295(18):2164-7.

2) Cho BY, Choi HS, Park YJ, Lim JA, Ahn HY, Lee EK, et al. Changes in the clinicopathological characteristics and outcomes of thyroid cancer in Korea over the past four decades. Thyroid 2013;23(7):797-804.

3) Lim H, Devesa SS, Sosa JA, Check D, Kitahara CM. Trends in thyroid cancer incidence and mortality in the United States, 1974-2013. JAMA 2017;317(13):1338-48.

4) Jeon MJ, Kim HK, Kim EH, Kim ES, Yi HS, Kim TY, et al. Decreasing disease-specific mortality of differentiated thyroid cancer in Korea: a multicenter cohort study. Thyroid 2018;28(9): 1121-7.

5) Haugen BR, Alexander EK, Bible KC, Doherty GM, Mandel SJ, Nikiforov YE, et al. 2015 American Thyroid Association management guidelines for adult patients with thyroid nodules and differentiated thyroid cancer: the American Thyroid Association guidelines task force on thyroid nodules and differentiated thyroid cancer. Thyroid 2016;26(1):1-133.

6) Yi KH, Lee EK, Kang HC, Koh Y, Kim SW, Kim IJ, et al. 2016 revised Korean Thyroid Association management guidelines for patients with thyroid nodules and thyroid cancer. Int J Thyroidol 2016;9(2):59-126.

7) Tufano RP, Teixeira GV, Bishop J, Carson KA, Xing M. BRAF mutation in papillary thyroid cancer and its value in tailoring initial treatment: a systematic review and meta-analysis. Medicine (Baltimore) 2012;91(5):274-86.

8) Xing M, Alzahrani AS, Carson KA, Viola D, Elisei R, Bendlova B, et al. Association between BRAF V600E mutation and mortality in patients with papillary thyroid cancer. JAMA 2013;309(14):1493-501.

9) Chen Y, Sadow PM, Suh H, Lee KE, Choi JY, Suh YJ, et al. BRAF(V600E) is correlated with recurrence of papillary thyroid microcarcinoma: a systematic review, multi-institutional primary data analysis, and meta-analysis. Thyroid 2016;26(2): 248-55.

10) Choi SY, Park H, Kang MK, Lee DK, Lee KD, Lee HS, et al. The relationship between the BRAF(V600E) mutation in papillary thyroid microcarcinoma and clinicopathologic factors. World J Surg Oncol 2013;11:291.

11) Xing M, Alzahrani AS, Carson KA, Shong YK, Kim TY,
Viola D, et al. Association between BRAF V600E mutation and recurrence of papillary thyroid cancer. J Clin Oncol 2015;33(1): $42-50$.

12) Jeong D, Jeong $Y$, Lee $S$, Lee $H$, Lee $W$, Kim $H$, et al. Detection of BRAF(V600E) mutations in papillary thyroid carcinomas by peptide nucleic acid clamp real-time PCR: a comparison with direct sequencing. Korean J Pathol 2012;46(1): 61-7.

13) Kwon MJ, Lee SE, Kang SY, Choi YL. Frequency of KRAS, $B R A F$, and PIK3CA mutations in advanced colorectal cancers: comparison of peptide nucleic acid-mediated PCR clamping and direct sequencing in formalin-fixed, paraffin-embedded tissue. Pathol Res Pract 2011;207(12):762-8.

14) Ito $Y$, Miyauchi $A$, Inoue $H$, Fukushima $M$, Kihara $M$, Higashiyama T, et al. An observational trial for papillary thyroid microcarcinoma in Japanese patients. World J Surg 2010;34(1):28-35.

15) Sugitani I, Toda K, Yamada K, Yamamoto N, Ikenaga M, Fujimoto Y. Three distinctly different kinds of papillary thyroid microcarcinoma should be recognized: our treatment strategies and outcomes. World J Surg 2010;34(6):1222-31.

16) Kim KJ, Kim SG, Tan J, Shen X, Viola D, Elisei R, et al. BRAF V600E status may facilitate decision-making on active surveillance of low-risk papillary thyroid microcarcinoma. Eur J Cancer 2020;124:161-9.

17) Walczyk A, Kowalska A, Kowalik A, Sygut J, Wypiorkiewicz $\mathrm{E}$, Chodurska R, et al. The BRAF(V600E) mutation in papillary thyroid microcarcinoma: does the mutation have an impact on clinical outcome? Clin Endocrinol (Oxf) 2014;80(6): 899-904.

18) Lee SM, Lee CR, Kang SW, Lee J, Jeong JJ, Nam KH, et al. Association between BRAFV ${ }^{600 E}$ mutations and clinicopathological features of papillary thyroid microcarcinoma (PTMC). J Endocr Surg 2019;19(3):76-84.

19) Nikiforova MN, Kimura ET, Gandhi M, Biddinger PW, Knauf JA, Basolo F, et al. BRAF mutations in thyroid tumors are restricted to papillary carcinomas and anaplastic or poorly differentiated carcinomas arising from papillary carcinomas. J Clin Endocrinol Metab 2003;88(11):5399-404.

20) Lin KL, Wang OC, Zhang XH, Dai XX, Hu XQ, Qu JM. The BRAF mutation is predictive of aggressive clinicopathological characteristics in papillary thyroid microcarcinoma. Ann Surg Oncol 2010;17(12):3294-300.

21) Zheng X, Wei S, Han Y, Li Y, Yu Y, Yun X, et al. Papillary microcarcinoma of the thyroid: clinical characteristics and BRAF(V600E) mutational status of 977 cases. Ann Surg Oncol 2013;20(7):2266-73.

22) Nam JK, Jung CK, Song BJ, Lim DJ, Chae BJ, Lee NS, et al. Is the BRAF(V600E) mutation useful as a predictor of preoperative risk in papillary thyroid cancer? Am J Surg 2012; 203(4):436-41.

23) Hong AR, Lim JA, Kim TH, Choi HS, Yoo WS, Min HS, et al. The frequency and clinical implications of the $B R A F(V 600 E)$ mutation in papillary thyroid cancer patients in Korea over the past two decades. Endocrinol Metab (Seoul) 2014;29(4):505-13.

24) $\mathrm{Li} \mathrm{X}$, Kwon $\mathrm{H}$. The impact of BRAF mutation on the 
BRAF Mutation in PTMC

recurrence of papillary thyroid carcinoma: a meta-analysis. Cancers (Basel) 2020;12(8):2056.

25) Guan H, Ji M, Bao R, Yu H, Wang Y, Hou P, et al. Association of high iodine intake with the T1799A BRAF mutation in papillary thyroid cancer. J Clin Endocrinol Metab 2009;94(5):1612-7.

26) Kim HJ, Park HK, Byun DW, Suh K, Yoo MH, Min YK, et al. Iodine intake as a risk factor for BRAF mutations in papillary thyroid cancer patients from an iodine-replete area. Eur J Nutr 2018;57(2):809-15.

27) Durante C, Puxeddu E, Ferretti E, Morisi R, Moretti S, Bruno $\mathrm{R}$, et al. BRAF mutations in papillary thyroid carcinomas inhibit genes involved in iodine metabolism. J Clin Endocrinol Metab 2007;92(7):2840-3.

28) Crispo F, Notarangelo T, Pietrafesa M, Lettini G, Storto G, Sgambato A, et al. BRAF inhibitors in thyroid cancer: clinical impact, mechanisms of resistance and future perspectives. Cancers (Basel) 2019;11(9):1388.

29) Hay ID. Management of patients with low-risk papillary thyroid carcinoma. Endocr Pract 2007;13(5):521-33.

30) Yu XM, Wan Y, Sippel RS, Chen H. Should all papillary thyroid microcarcinomas be aggressively treated? An analysis of 18,445 cases. Ann Surg 2011;254(4):653-60. 